\title{
Thermal stability and temperature dependent electron spin resonance characteristics of the As acceptor in geological $2 \mathrm{H}-\mathrm{MoS}_{2}$
}

\author{
B Schoenaers, A Stesmans and V V Afanas'ev \\ E-mail: andre.stesmans@kuleuven.be \\ Received 9 October 2018, revised 9 January 2019 \\ Accepted for publication 21 January 2019 \\ Published 19 February 2019 \\ Abstract
}

Department of Physics and Astronomy, University of Leuven, B-3001 Leuven, Belgium

Electron spin resonance (ESR) results are presented on the thermal stability and temperature dependence of the ESR spectral characteristics of the As acceptor dopant (As substituting for $\mathrm{S}$ site) in geological 2H-MoS2. Under sequential isochronal heating in $\mathrm{H}_{2}$ (1.1 atm), the As dopant density is found to remain unaffected for anneal temperatures ( $\mathrm{Tans}$ ) up to $525^{\circ} \mathrm{C}$, above which the density moderately decreases ( $\sim 3$ times) for $\mathrm{T}_{\mathrm{an}} \rightarrow \cdot 840^{\circ} \mathrm{C}$. In turn, vacuum annealing is seen to result in a gradual increase (2-3 times) of the As acceptor density for Tan increasing from $400{ }^{\circ} \mathrm{C} \cdot \rightarrow \cdot 840^{\circ} \mathrm{C}$, pointing to a positive 'regain' of misconfigured As impurities in pristine geo-MoS2. Finally, meticulous monitoring of the ESR signal intensity versus tempertaure at $\mathrm{X}$-band confirms the previously inferred As acceptor activation energy of $0.7 \cdot \pm \cdot 0.2 \mathrm{meV}$ at K-band. The appropriateness of the latter ESR probing and analysis approach has been consolidated by application to the archetypal case of decoupled $\mathrm{P}$ donors in $\mathrm{n}$-Si, resulting in an ESR-determined activation energy $(42 \cdot \pm \cdot 4 \mathrm{meV})$ well in agreement with the generally accepted one. In light of these findings, As is confirmed as a promising candidate for stable covalently bonded p-type doping of $\mathrm{MoS}_{2}$.

Keywords: molybdenum disulfide, As acceptor, dopant activation energy, EPR, substitutional p-type doping, thermal stability of dopants

(Some figures may appear in colour only in the online journal)

1. Introduction

Propelled by the discovery of graphene [1], two dimensional

(2D) transition metal dichalcogenides (TMDs) have emanated as promising candidates to revolutionize the current semiconductor technology as they could potentially replace conventional semiconductors in future nanoelectronic devices [2-8]. Among the comprehensive family of TMDs, molybdenite $\left(\mathrm{MoS}_{2}\right)$ has been widely studied for its outstanding properties: it is composed of layered stacks of hexagonal covalently bonded S-Mo-S unit sheets, forming an atomically thin, strong, and flexible layered semiconductor with an advantageous direct band gap of $\sim 1.85 \mathrm{eV}$ in monolayer form and an indirect band gap of $\sim 1.3 \mathrm{eV}$ in bulk form $[2,3]$. Owing to its unique characteristics, MoS2 is particularly well suited for incorporation in innovative integrated circuits and optoelectronic devices like e.g. photodiodes, light-emitting diodes, and flexible and transparent electronics [2, 3]. Additionally, the interlayer van der Waals bonding character of the 
2D sheets potentially allows for multi-material stacking with other 2Ds that are characterized by different mechanical, electrical, and optical properties [9]. This novel approach would enable the creation of advanced heterostructure devices with no lattice mismatch issues and unprecedented functionalities.

While the potential of TMDs, and MoS2 in particular, to be incorporated in a variety of future devices seems promising, the production of high quality large area layers still presents itself as a major challenge. Similar to other layered materials, pristine $\mathrm{MoS}_{2}$ flakes can be obtained by micromechanical exfoliation, resulting in the current highest

mobility $\mathrm{MoS}_{2}$-based devices $[4,10,11]$. However, not

Semiconductor Science and Technology

Semicond. Sci. Technol. 34 (2019) 035022 (6pp) https://doi.org/10.1088/1361-6641/ab004f $0268-1242 / 19 / 035022+06 \$ 33.001$ @ 2019 IOP Publishing Ltd Printed in the UK

different from other production methods like e.g. chemical vapor deposition (CVD), sulfurization and atomic layer deposition, exfoliated flakes suffer from the presence of impurities as well as native defects which hamper electronic transport properties. Therefore, a thorough understanding of the role of defects and impurities is of key importance for intended applications.

Evidently, besides reliable production, full exploitation of the unique properties of $\mathrm{MoS}_{2}$ as atomically thin semiconductor material in novel devices requires reliable, well controlled doping, preferably achieved by stable covalent bonding of substitutional impurities. Yet, the current essential knowledge on dopant characteristics, including its nature, concentration, thermal stability and, crucially, corresponding energy level positioning within the $\mathrm{MoS}_{2}$ band gap, is still limited.

Albeit with fluctuating awareness, electrical doping of MoS2 has not been left unattended in the past. However, the interest in understanding and introducing controlled, stable doping of $\mathrm{MoS}_{2}$ - both theoretically and practically-has accelerated steadily over the recent years, obviously propelled by the endeavor after 2D applications (see, e.g. [12] for a brief synopsis and references therein). Both doping by intrinsic defects and (substitutional) impurities have been addressed. As characterized, and mostly identified, by electron spin resonance (ESR) - the method of the current work-, the latter category includes Re (substituting for Mo) in 2D MoS2, operating as a donor [13], as well as the acceptors $\mathrm{Zr}, \mathrm{Nb}, \mathrm{As}$, and $\mathrm{V}$ in 3R-MoS2 grown by chemical vapor transport $[14,15]$, and As $[14,16]$ in natural (geological) $2 \mathrm{H}-\mathrm{MoS}_{2}$ and $\mathrm{N}[12,17,18]$ in synthetic CVD-grown $2 \mathrm{H}-\mathrm{MoS} 2$. Also the $\mathrm{Pb}$ impurity, likely substituting for Mo and operating as acceptor, has been tentatively assigned by ESR [19]. Only recently, the thermal activation energy, $E_{a}$, of impurity dopants has been exclusively and atom-specifically assessed by ESR, reporting for $2 \mathrm{H}-\mathrm{MoS}_{2}$ the values $\mathrm{Ea}_{\mathrm{a}}$ $=\cdot 0.7 \cdot \pm \cdot 0.2 \mathrm{meV}$ and $45 \cdot \pm \cdot 7 \mathrm{meV}$ for As [16] and $\mathrm{N}$ [17], respectively, with a lower bound value, $\mathrm{E}_{\mathrm{a}}>\cdot 10 \mathrm{meV}$, being 
estimated for $\mathrm{Pb}[19]$.

The current work reports and expands on the identification by a multi-frequency ESR study of the As acceptor in

geological 2H-polytype MoS2, with the As impurities substituting for $\mathrm{S}$ sites. Careful monitoring of the ESR signal

intensity over a broad temperature $(T)$-range previously

revealed the As dopant as a very shallow acceptor with activation

energy $\mathrm{Ea}_{\mathrm{a}} \cdot \approx \cdot 0.7 \mathrm{meV}$ [16].

Progressing on these findings, this work presents a detailed analysis of the thermal stability and reactivity of the As acceptor under heat treatment in $\mathrm{H}_{2}$ and vacuum, including a comparison with the $\mathrm{N}$ acceptor in synthetic $2 \mathrm{H}-\mathrm{MoS} 2$ [12]. Additionally, the As acceptor's activation energy has been separately extracted from X-band ESR to verify the initial assessment based on K-band observations [16]. Finally, to consolidate the latter applied probing and interpretation approach, the activation energy of the $\mathrm{P}$ donor in $\mathrm{n}-\mathrm{Si}$ is obtained from K-band ESR measurements and compared to the commonly accepted value of $\sim 45 \mathrm{meV}$ [20].

2. Experimental details The material studied in this work is a commercially obtained [21] bulk geological MoS2 crystal of 2H-polytype (hexagonal symmetry, two MoS2 layers per repeat unit and Mo in trigonal prismatic coordination bonded to $6 \mathrm{~S}$ atoms). A thermoelectric test demonstrated the sample to be manifest p-type, as also evidenced by the observed As acceptor ESR spectra. Thin ( 0.2-0.4 mm) slices of $\sim 2 \cdot \times \cdot 8 \mathrm{~mm} 2$ main area were cut from the parent MoS2 crystal, with the c-axis along the main face normal $n$, appropriate for ESR study.

ESR observations were carried out over the T-range $1.5-40 \mathrm{~K}$ by means of standard continuous wave absorption X-band $(\sim 8.9 \mathrm{GHz})$ and $\mathrm{K}$-band $(\sim 20.4 \mathrm{GHz})$ spectrometers. First derivative spectra were recorded using harmonic modulation (Bmcos $(\omega m \mathrm{t})$, with $\omega_{\mathrm{m}} / 2 \pi \cdot \approx \cdot 100 \mathrm{kHz}$ ) of the applied magnetic field $B$.

Essentially, both observational frequencies may be equally well applied for reliable analysis of the As ESR signal in all its aspects. However, K-band observations are here routinely performed because of the intrinsically higher sensitivity of this locally constructed spectrometer than the $\mathrm{X}$-band one. The latter is involved in this work to independently verify key defect parameters, such as the acceptor activation energy, initially inferred from K-band work, or, in some cases, to facilitate optimal sample mounting in the cavity.

For each specific investigation of ESR signal intensityversustemperature behavior, ESR observations were generally made either for $\mathrm{B} / / \mathrm{C}$-axis or $\mathrm{B} \perp \mathrm{c}$-axis, with $\mathrm{B}$ uniformly (relative field inhomogeneity $<1.5 \cdot x \cdot 10-6$ over sample size) applied over the entire sample. As it is known the As acceptor ESR signal in $\mathrm{MoS}_{2}$ to show profound $\mathrm{g}$ anisotropy (see, e.g. $[15,16])$, this ensures measurement of 
'identical' spectra (with respect to g) over the entire case study.

In aiming for accurate determination of defect (acceptor) densities - of prime interest here-and g values, use was made of two co-mounted isotropic marker signals (samples),

i.e. Si:P $[\mathrm{g}(4.3 \mathrm{~K}) \cdot=\cdot 1.99869 \cdot \pm \cdot 0.00002$; $\operatorname{spin} \mathrm{S} \cdot=\cdot 1 / 2]$ [22]

and LiF: Li (g $\cdot=\cdot 2.002293 \cdot \pm \cdot 0.000002 ; \mathrm{S} \cdot=\cdot 1 / 2$ ) [23], both

well calibrated in terms of absolute number of spin centers included and g value. Generally, both markers may in principle serve equally well for assisting in analysis of a studied ESR signal; preference of one before the other for use in a specific study just stems from considerations about 'opportunistic' spectroscopic adequacy, such as avoiding signal overlap (making use of difference in g value), line width considerations, etc. The defect density is derived from the ESR signal intensity, obtained as the double numerical integration of the detected $\mathrm{dP} \mu \mathrm{r} / \mathrm{dB}$ signal, where $\mathrm{P}_{\mu \mathrm{r}}$ is the reflected microwave power. Though not a major subject here, the calibrated $\mathrm{g}$ markers optimally allowed for accurate $\mathrm{g}$ value monitoring. In line with previous findings [16], the As ESR signal g value is observed to remain constant within experimental accuracy over the whole observational T-range covered, and no change is noticed as a function of anneal temperature either. In experimenting, attention was paid to 2 Semicond. Sci. Technol. 34 (2019) 035022 B Schoenaers et al avoid spectral signal distortion through careful control of spectrometer settings, i.e. appropriately restricted magnitudes of $\mathrm{Bm}_{\mathrm{m}}$ and incident microwave power $\mathrm{P}_{\mu}$ (see, e.g. [24] for a general outline of the effects of (over)modulation and microwave saturation on the spectral appearance). In the current work, values were limited as: $\mathrm{Bm} \cdot \cdot 0.5 \mathrm{G}$ (both Xand

K-band), $\mathrm{P}_{\mu}(\mathrm{X}$-band) $\cdot \cdot 0.3 \mu \mathrm{W}$, and

$\mathrm{P}_{\mu}(\mathrm{K}$-band $) \cdot \cdot 8 \mathrm{nW}$ for $\mathrm{T} \cdot \cdot 10 \mathrm{~K}$ and $\mathrm{P}_{\mu}(\mathrm{K}$-band $) \cdot \cdot 25 \mathrm{nW}$

for $10 \mathrm{~K} \cdot \cdots \cdot \mathrm{T} \cdot 40 \mathrm{~K}$.

As for the current geological MoS2 material studied, the As dopant density in the as-received sample has been determined by ESR as $\sim 6 \cdot \times \cdot 1017 \mathrm{~cm}-3$, the value remaining unaltered after reference setting thermal treatment in vacuum $\left(\sim 400^{\circ} \mathrm{C}\right)$. As outlined previously [16], the measurements have been carried out at sufficiently low $\mathrm{T}(\sim 2 \mathrm{~K})$ where the As dopants reside in their electrically non-activated (i.e. ESRactive) state, thus ensuring assessment of the full As acceptor system.

3. Results and discussion

3.1. Dopant thermal stability

Aiming at a better understanding of the dopant stability and reactivity, the As acceptor has been subjected to two series of thermal treatments, one performed in $\mathrm{H}_{2}(1.1 \mathrm{~atm})$, the other in high vacuum $\left(<7 \cdot \times \cdot 10_{-6} \mathrm{mbar}\right)$. Isochronal thermal steps ( $\sim 5 \mathrm{~min}$ ) were performed sequentially on two pieces of the 
same sample, priorly subjected to a 'reference' setting treatment in vacuum $\left(\sim 400{ }^{\circ} \mathrm{C} ; \sim 21 \mathrm{~h}\right)$, referred to as the initial state. Thermal treatments were performed using a conventional resistively heated furnace system foreseen with a double-walled high-purity quartz insert, as described elsewhere [25].

The main purpose of the thermal treatment in $\mathrm{H}_{2}$ is to investigate possible inactivation (passivation) of the acceptors through defect-H bond formation, inspired by the passivation behavior of some dopants in other semiconductors (see, e.g. [26]). Afterwards, the sample is subjected to subsequent anneals in vacuum in an attempt to get a better understanding of the reactivation (defect-H bond dissociation) of the acceptors (see, e.g. [27] and references therein). Concurrently, the extensive thermal treatment investigation also provides valuable insight into the overall structural thermal stability of the substitutional acceptor impurities.

Figure 1 shows the evolution of the As ESR signal intensity, which is proportional to the non-activated As acceptor density, over the various thermal treatments. Looking at the data corresponding to the vacuum thermal treatments, a first trend is revealed: starting from its reference value observed after heating in vacuum at an anneal temperature $\mathrm{T}_{\mathrm{an}}=\cdot 400^{\circ} \mathrm{C}$, the signal intensity increases gradually for subsequent thermal steps Tans up to $840^{\circ} \mathrm{C}$ likely tending to reach a plateau at a $\sim 2.5$ times higher value for Tan

$$
\text { - } 700^{\circ} \mathrm{C} \text {. }
$$

In studying a geological $\mathrm{MoS}_{2}$ specimen, one may advance various interpretations for this effect. In a first view one may suggest it to result from structurally 'ill configured' As atoms moving to the 'proper' dopant position under thermal stimulus. Alternatively, it may concern a defect reactivation effect, e.g. defect-H bond breaking, or, perhaps better, defect 'decomplexation' in general: as exposed [6], geological $\mathrm{MoS}_{2}$ is known to be besieged by a multitude of different impurities, some of which may tie up with As.

Anyway, in both views, the general message is that the As impurity concentration actually present is higher than that corresponding to the initially observed As acceptor concentration, and additional As impurities can be regained for the proper acceptor doping bath by thermal treatment. As to the thermal treatment in $\mathrm{H}$, the As signal is seen to remain unaffected, within experimental accuracy, for heating up to $\sim 525^{\circ} \mathrm{C}$, to then gradually be decreased by a factor of three at $\mathrm{T}_{\text {an }} \approx \cdot 840^{\circ} \mathrm{C}$. The latter behavior is in contrast with that of the $\mathrm{N}$ acceptor in synthetic MoS2 [12] which experiences a drastic ESR intensity drop by an order of magnitude at $\mathrm{T}_{\mathrm{an}} \approx \cdot 500{ }^{\circ} \mathrm{C}$. Yet, for both acceptors, the intensity decrease can be tentatively ascribed to the direct passivation (inactivation) of the respective acceptors through defect- $\mathrm{H}$ bond (or defect-H complex) formation, similar to previous results for inactivation of point defects, such as, e.g. boron 
acceptors in Si [26]. Notably, the apparent gradual intensity decrease of the As acceptor points to a significantly higher activation energy for passivation when compared to the $\mathrm{N}$ acceptor which experiences a more abrupt intensity drop in the Tan-range $\sim 370{ }^{\circ} \mathrm{C}-500{ }^{\circ} \mathrm{C}[12]$.

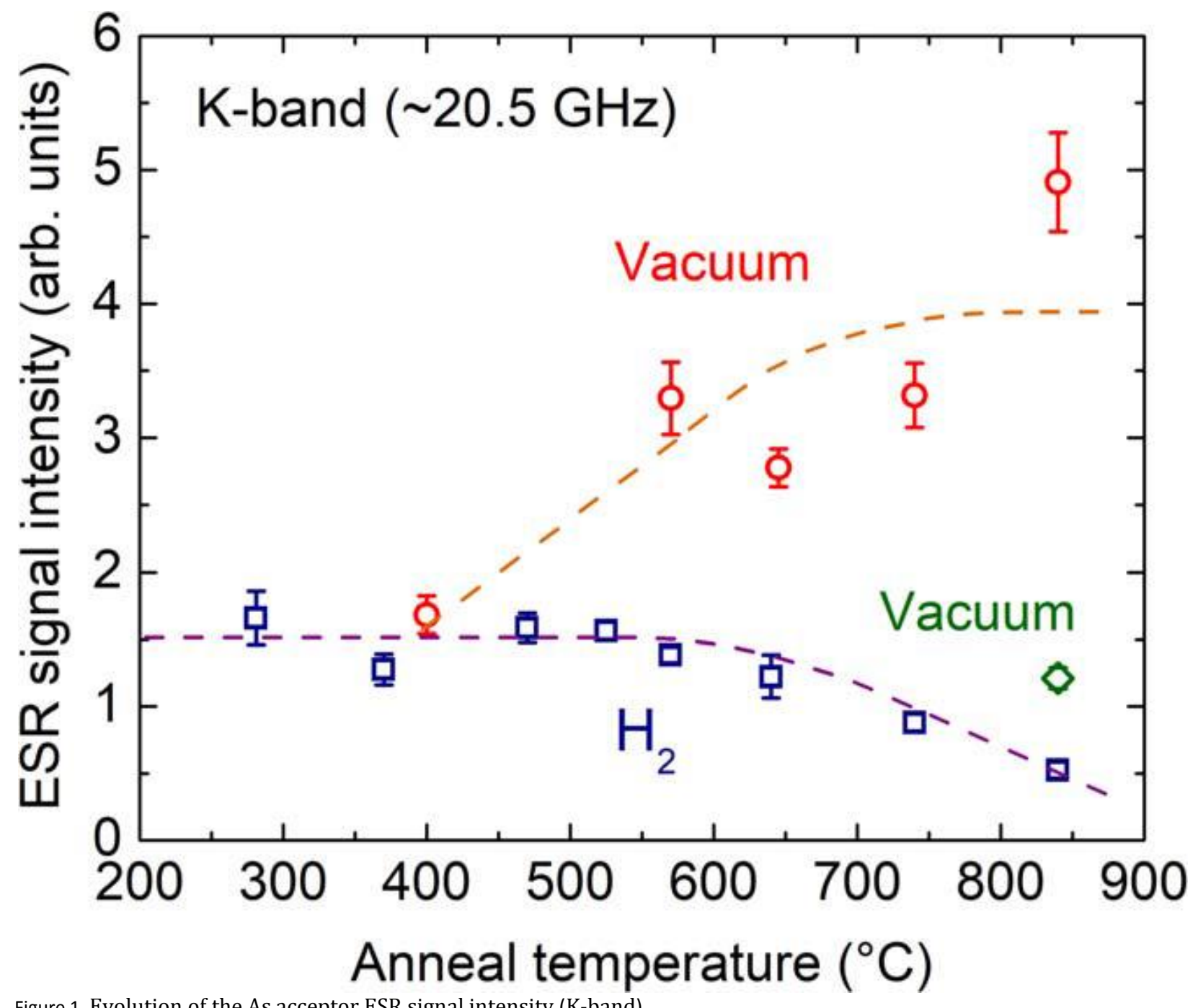

Figure 1. Evolution of the As acceptor ESR signal intensity (K-band) as a function of isochronal heating in $\mathrm{H}_{2}$ (blue squares) and vacuum (red circles). ESR measurements were carried out at $\mathrm{T} \cdot \approx \cdot 4.2 \mathrm{~K}$ for $\mathrm{B} \perp \mathrm{n}$ using $\mathrm{Bm}_{\mathrm{m}}=\cdot 0.5 \mathrm{G}$ and $\mathrm{P}_{\mu}=\cdot 25 \mathrm{nW}$. The signal intensity increases gradually after sequential thermal treatments in vacuum and appears not affected in $\mathrm{H}_{2}$ up to $\sim 525^{\circ} \mathrm{C}$. The signal intensity decreases after thermal steps in $\mathrm{H}_{2}$ by a factor of about three from $\sim 525^{\circ} \mathrm{C}$ to $\sim 840{ }^{\circ} \mathrm{C}$ to be only partially recovered after a vacuum anneal at $840{ }^{\circ} \mathrm{C}$ (green diamond). The decrease of the signal intensity in $\mathrm{H}_{2}$ is tentatively ascribed to passivation of the As acceptors by bonding to $\mathrm{H}$. Error bars denote the uncertainty [(maximum value - minimum value $) / 2]$ for the set of data taken at a specific temperature. The dashed curves guide the eye.

3

Semicond. Sci. Technol. 34 (2019) 035022 B Schoenaers et al 
Additionally, contrary to the case of the inactivated $\mathrm{N}$ acceptors that cannot be reactivated at temperatures up to $\sim 740^{\circ} \mathrm{C}$ [12], (partial) reactivation of the As acceptor signal intensity by approximately a factor of two does occur at $\mathrm{Tan}$ $=\cdot 840^{\circ} \mathrm{C}$ in vacuum (see figure 1 ). The latter would indicate that the activation energy for reactivation of the As acceptors is significantly higher than the corresponding activation energy (Eact $\cdot 3.3 \mathrm{eV}$ ) for the $\mathrm{N}$ acceptor estimated in previous work [12].

3.2. Temperature dependence

3.2.1. Dopant activation energy. Besides serving as an exclusive technique for atomic identification and thermal stability assessment of dopant impurities, ESR also provides the unique possibility to reliably determine the dopant-type specific activation energy through monitoring versus $T$ the ESR signal intensity which is proportional to the concentration of non-activated acceptors. The acceptor activation energy, denoted as $E_{a}=\cdot E_{A}-E_{v}$ with $E_{A}$ the acceptor energy level and Ev the valence band (VB) maximum, can then be obtained by interpreting the data using the proper mathematical expression (equation (1)) derived within the standard semi-classical solid state theory of band structure for well-defined conditions, as discussed before $[16,28]$.

For the current purpose, the As acceptor signal is monitored for $\mathrm{B} / / \mathrm{c}$-axis using $\mathrm{X}$-band ESR, figure 2 showing a representative spectrum $(\mathrm{g} / / \cdot=\cdot 2.027(1))$ observed at $\mathrm{T} \cdot=\cdot 4.2 \mathrm{~K}$, together with the co-mounted LiF:Li marker signal $(\mathrm{g} \cdot=\cdot 2.00229)$. Figure 3 shows the attained normalized ESR intensity-versus-T behavior of the 75As hyperfine (hf) quartet signal after proper correction for the admitted Curie-type susceptibility $\chi \propto 1 / T$-dependence of the spin system's specific magnetism [16]. As demonstrated by the dashed curve, i.e. plot of equation (1) for $E_{a}=\cdot 0.7 \mathrm{meV}$, the data reliably confirm the previously obtained strong activationdriven signal intensity decay of the As acceptor at $\mathrm{K}$-band, where from the latter data, the value $\mathrm{E}_{\mathrm{a}}$ $=\cdot 0.7 \cdot \pm \cdot 0.2 \mathrm{meV}$ had been inferred [16]. The data are interpreted (see dashed curve in figure 2) using the expression for the ratio of neutral, ESR-active, $N_{\mathrm{A}}{ }^{*}$, to total, $\mathrm{N}_{\mathrm{A}}$, acceptor densities derived from dopant occupation statistics $[16,28]$

*

$=-$

$++$

()

$N$ 


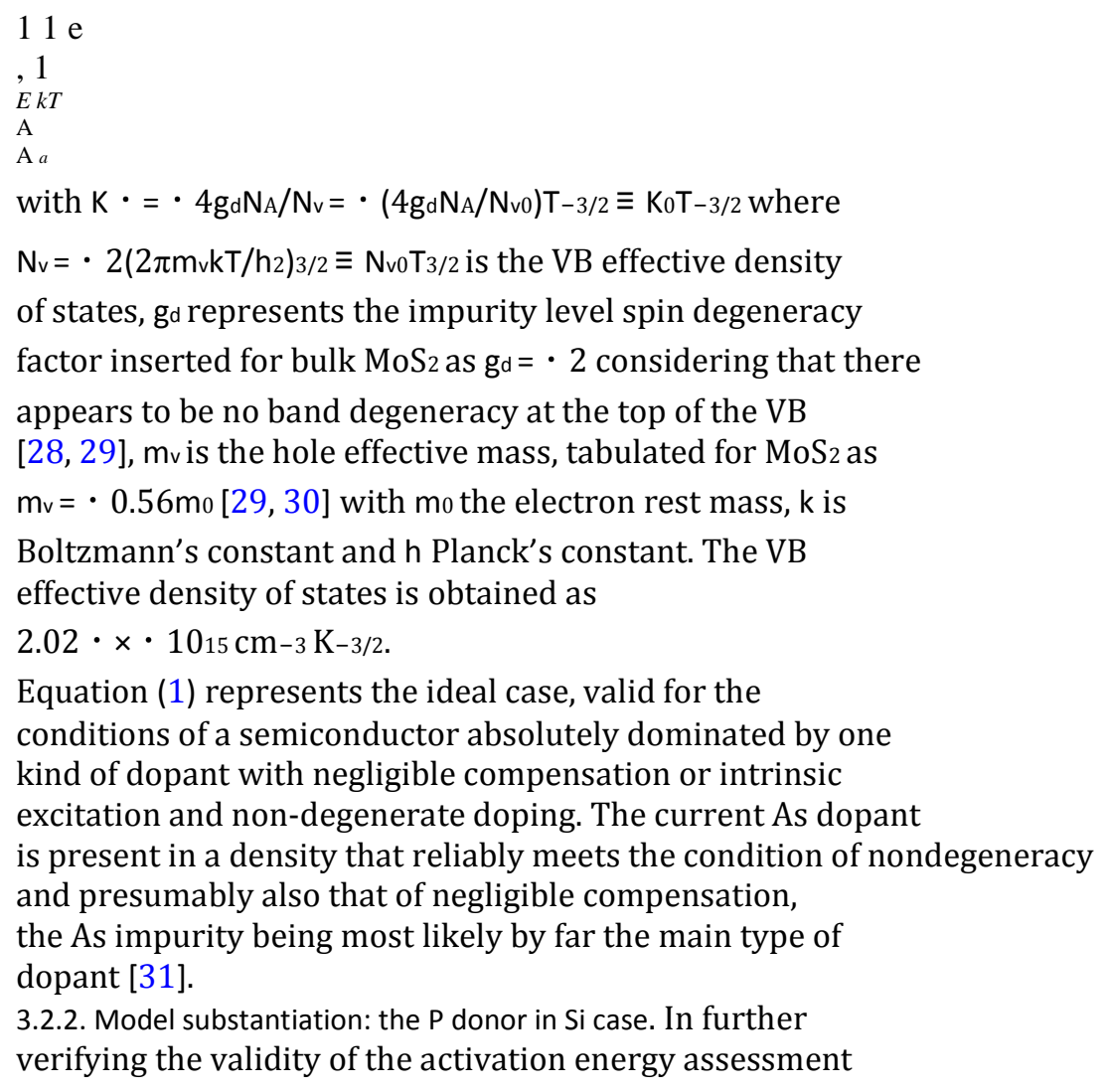




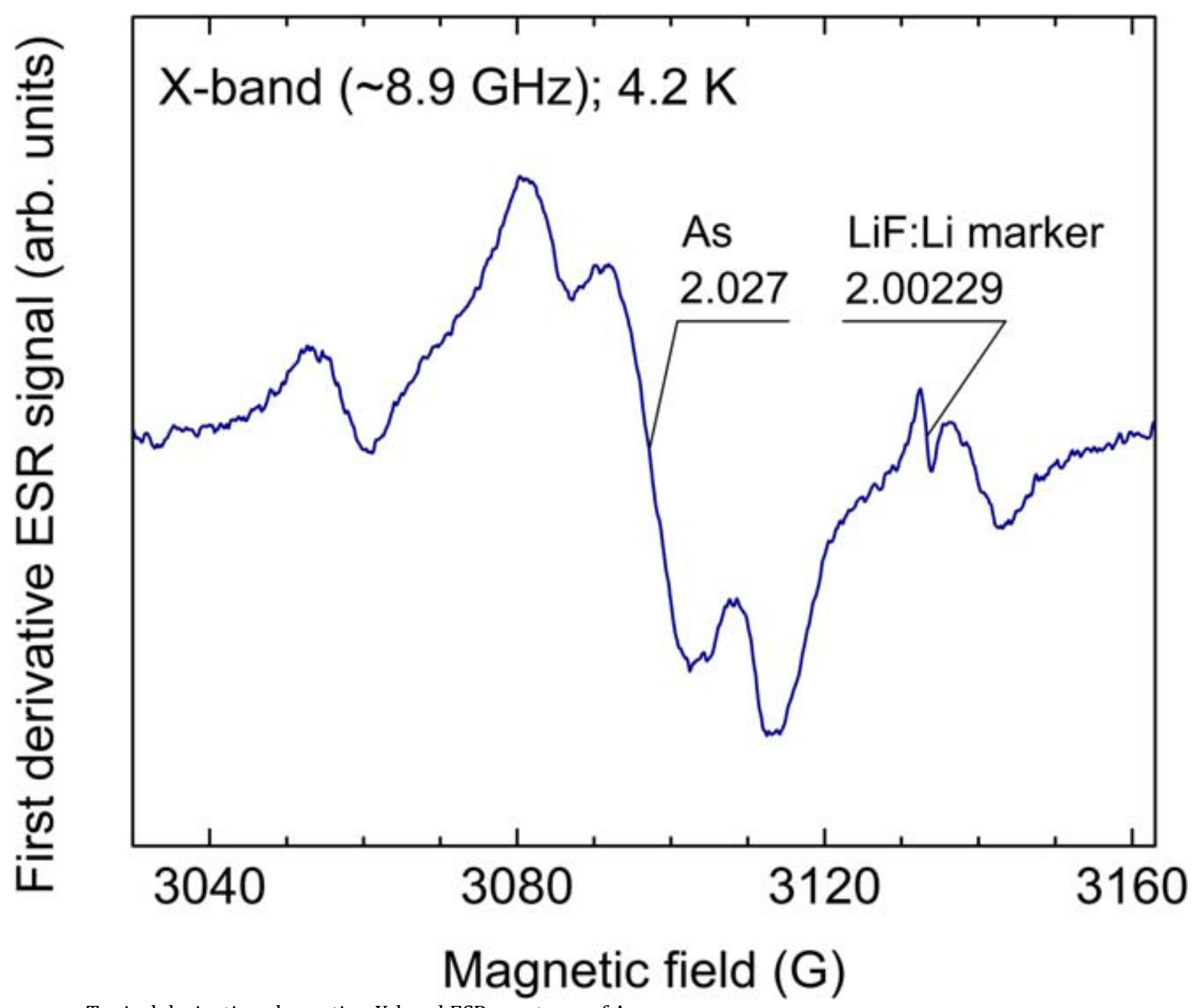

Figure 2. Typical derivative absorption X-band ESR spectrum of As acceptor impurities in bulk geological $2 \mathrm{H}-\mathrm{MoS}_{2}$ observed for $\mathrm{B} / / \mathrm{n}$

at $4.2 \mathrm{~K}$ using incident microwave power $\mathrm{P}_{\mu} \approx \cdot 0.3 \mu \mathrm{W}$ and $\mathrm{Bm}$

$=\cdot 0.5 \mathrm{G}$. The narrow signal at $\mathrm{g} \cdot=\cdot 2.00229$ stems from the comounted LiF:Li marker sample. 


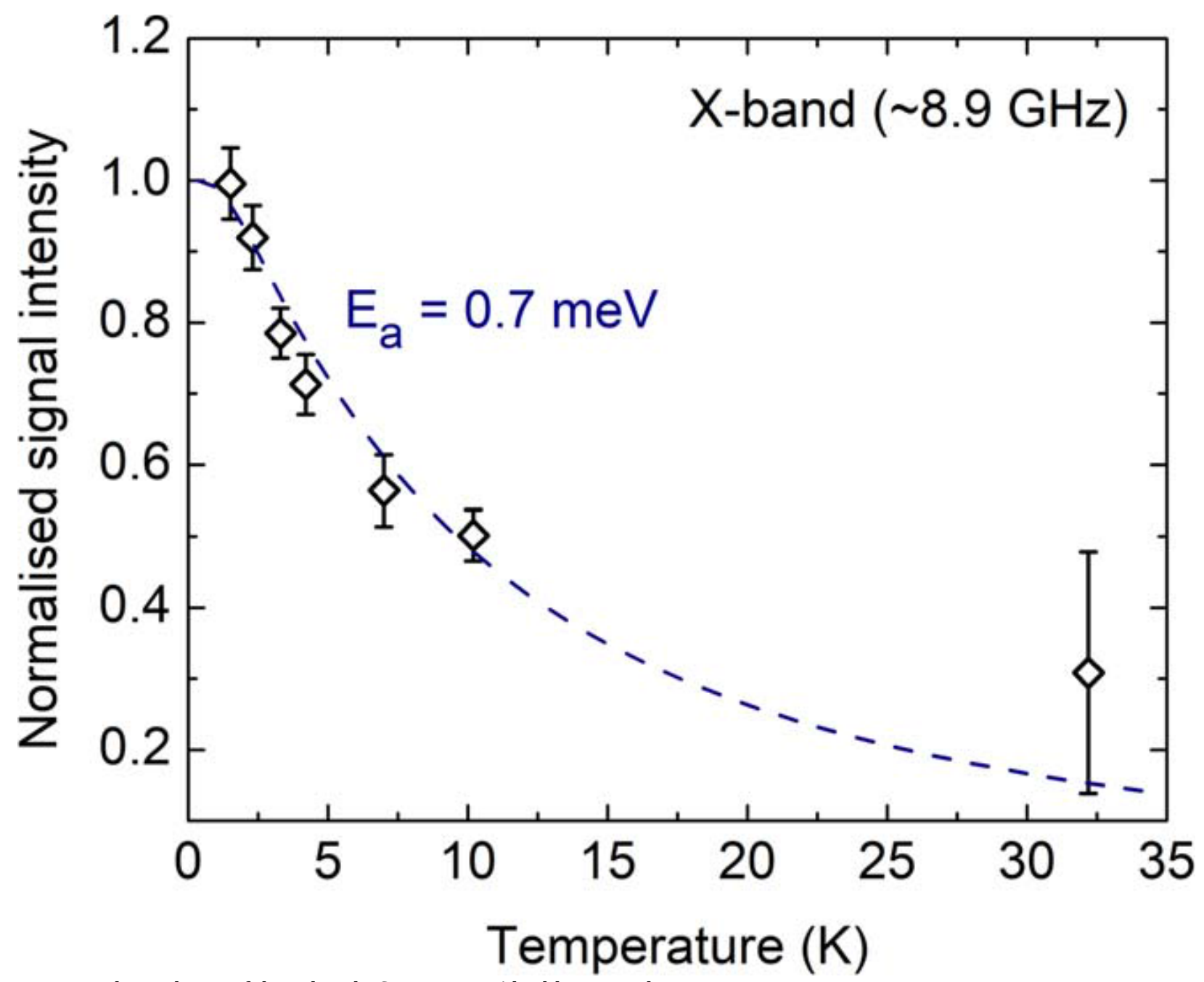

Figure 3. T-dependence of the X-band ESR intensity (double integral of derivative absorption spectra; B//c-axis) of the 75As hf ESR pattern originating from single decoupled As acceptors in geological $2 \mathrm{H}-\mathrm{MoS} 2$. The data, recorded over the T range 1.6-32 K, are normalized to the low $\mathrm{T}$ plateau value ( $\mathrm{T} \cdot \cdot 1.6 \mathrm{~K}$ ) after correction for the admitted $\chi \propto 1 / T$-dependence. The dashed curve represents equation (1) plotted with $E_{a}=\cdot 0.7 \mathrm{meV}$. The error bars represent the uncertainty within data sets.

Semicond. Sci. Technol. 34 (2019) 035022 B Schoenaers et al based on employing equation (1) to analyze the ESR dopant intensity versus T behaviour, the current interpretation approach has been independently tested on a sample taken from an n-type (100)Si wafer P-doped to a density of, as determined by ESR, $[\mathrm{P}] \cdot=\cdot(1.0 \cdot \pm \cdot 0.1) \cdot \times \cdot 10_{17} \mathrm{~cm}-3$, well in agreement with the value $9 \cdot \times \cdot 1016 \mathrm{~cm}-3$ specified by the supplier on the basis of conductivity data. In illustrating the subject of study, figure 4 shows a typical X-band spectrum $\left(\mathrm{T} \cdot=\cdot 22.5 \mathrm{~K}\right.$ ) of isolated ${ }_{31} \mathrm{P}$ donors (substituting for $\mathrm{Si}$ ) in c-Si, appearing as the well-known iso-shaped $\sim 41 \mathrm{G}$ split doublet, (see, e.g. [32]) together with the signal of the comounted 
Si:P marker $(\mathrm{g} \cdot=\cdot 1.9987)$.

Similar to the As acceptor, the P donor most likely meets the same conditions required for the proper application of equation (1), i.e. non-degenerate doping dominated by one kind of dopant and negligible compensation. Figure 5 presents the temperature dependence of the normalized $\mathrm{X}$-band ESR signal intensity corresponding to the ${ }_{31} \mathrm{P} \mathrm{hf}$ doublet stemming from single decoupled $\mathrm{P}$ dopants in $\mathrm{Si}$. Optimized fitting of equation (1) to the data shown in figure 5 results in $\mathrm{E}_{\mathrm{a}}=\cdot 42 \cdot \pm \cdot 4 \mathrm{meV}$, well in agreement with the commonly accepted value of $\sim 45 \mathrm{meV}$ [20], thus substantiating the interpretation model outlined in the previous paragraph.

3.2.3. As clusters. As described before [16], only $13 \%$ of the total As spectrum is found to be composed of the 75As fourpeak hf structure, corresponding to dopants residing in the preferred 'isolated' doping state, while the major part of the spectrum $(87 \%)$ has been attributed to exchange-coupled As acceptors denoted as 'cluster' formation. In the context of the process of the determination of the dopant Ea through ESR signal intensity monitoring, it may thus be valuable to closer verify the T-dependence of the latter part of the ESR spectrum.

Figure 6 shows a least-square linear fitting of the inverse signal intensity of the total As spectrum versus $\mathrm{T}$, exposing a paramagnetic $\left(\chi \cdot \propto \cdot\left(T \cdot-\cdot T_{c}\right)-1\right)$ behavior with a close to zero Curie-Weiss temperature $\mathrm{Tc}=\cdot-1 \cdot \pm \cdot 1 \mathrm{~K}$, similar to the results found for other dopants in Si [22,33]. This clearly demonstrates the limited influence of the genuine intensity 


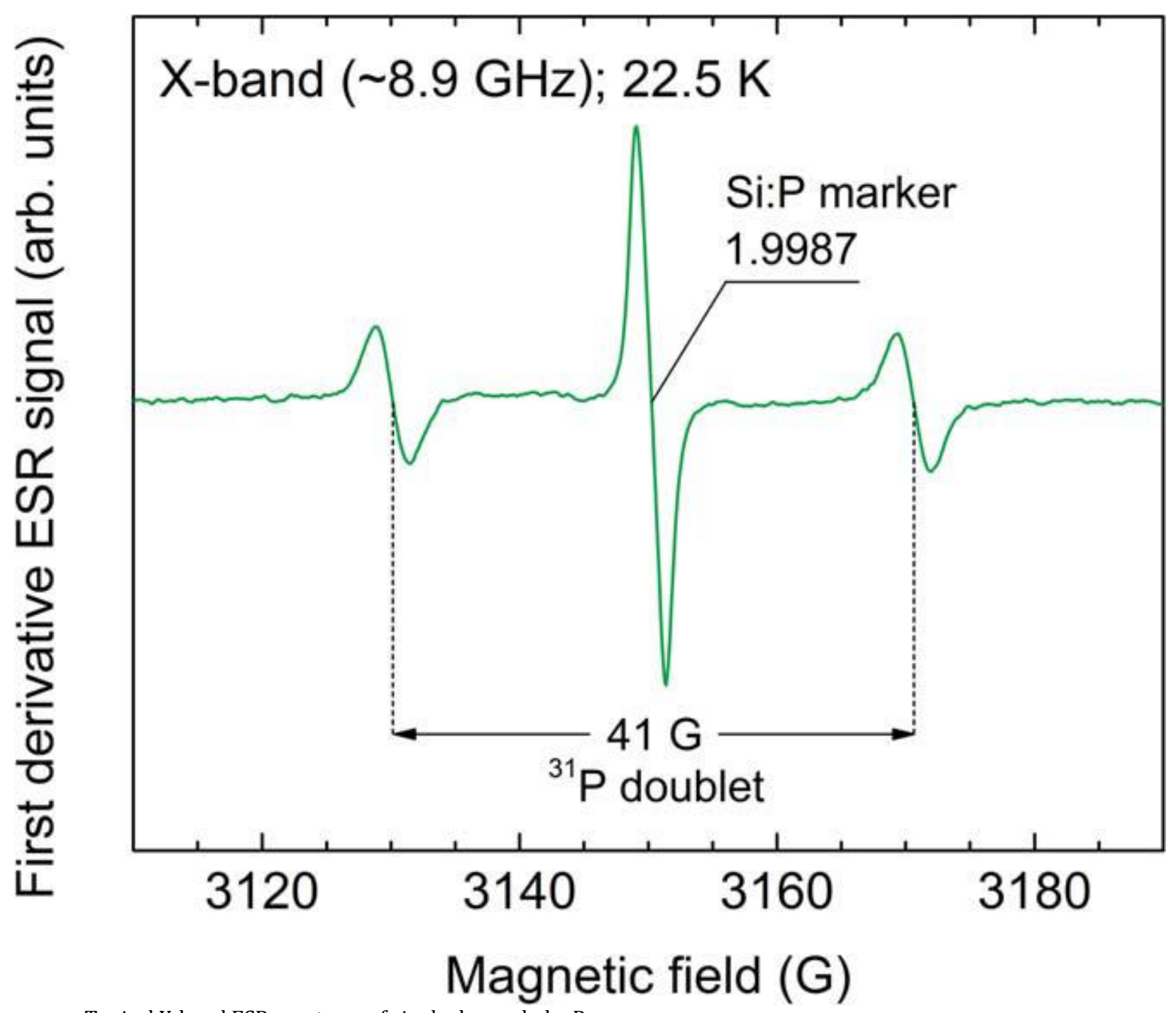

Figure 4. Typical X-band ESR spectrum of single decoupled ${ }_{31} \mathrm{P}$ donors substituting for $\mathrm{Si}$ in n-type c-Si observed at $22.5 \mathrm{~K}$. The dashed lines mark the $\sim 41 \mathrm{G}$ splitting of the well-known iso-shaped ${ }_{31} \mathrm{P}$ hf doublet. The central signal at $\mathrm{g} \cdot=\cdot 1.9987$ stems from the comounted Si:P marker sample. 


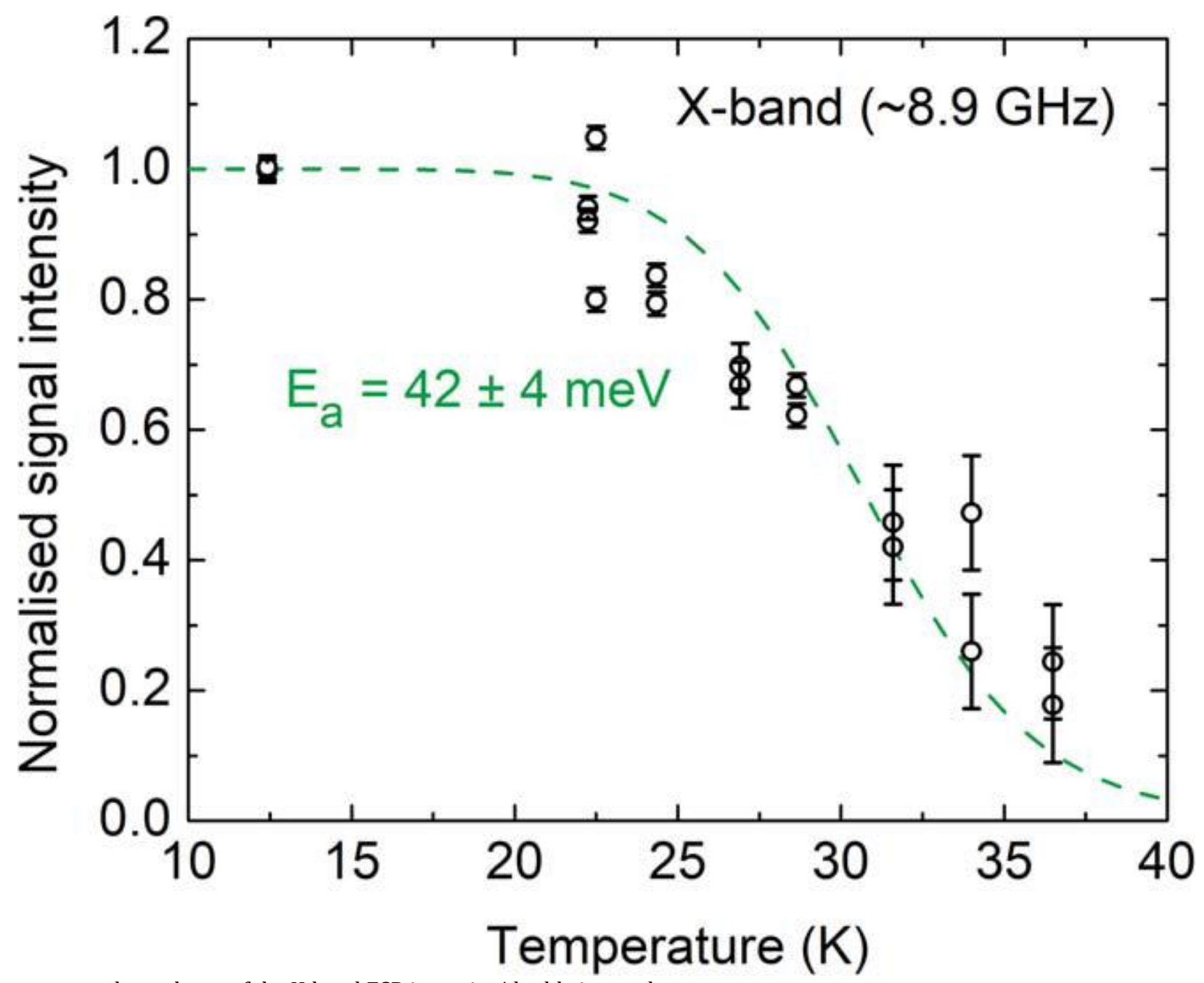

Figure 5. T-dependence of the K-band ESR intensity (double integral of derivative absorption spectra) of the isotropic ${ }_{31} \mathrm{P}$ hf doublet stemming from single decoupled P dopants in n-type c-Si. Optimized fitting of equation (1), represented by the dashed curve, results in an activation energy $\mathrm{E}_{\mathrm{a}}=\cdot$ $42 \cdot \pm \cdot 4 \mathrm{meV}$ which is in excellent agreement with the commonly accepted value of $\sim 45 \mathrm{meV}$, [20] providing confidence in the applied interpretation approach (see equation (1)). The error bars reflect the uncertainty within data sets. 


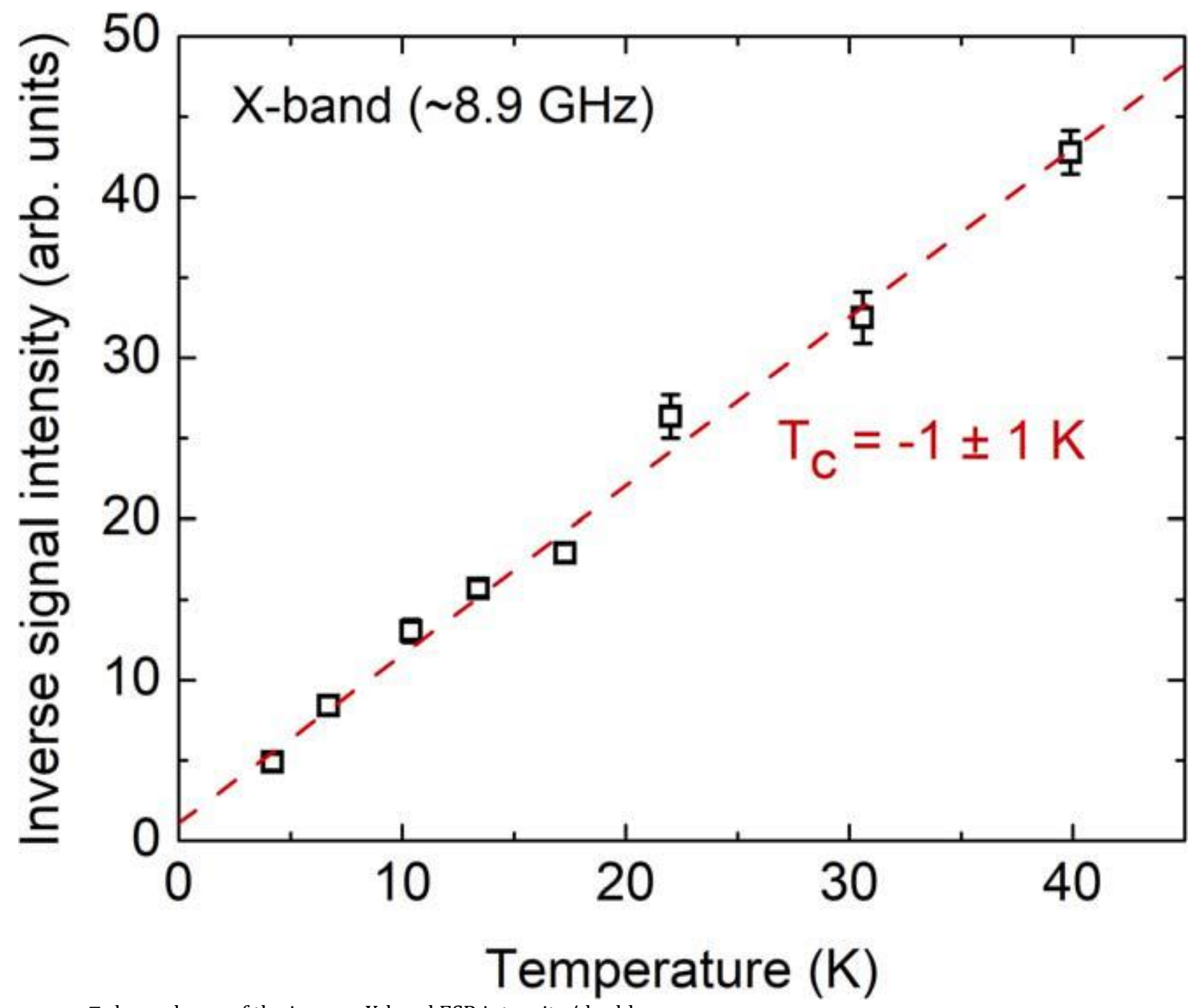

Figure 6. T-dependence of the inverse X-band ESR intensity (double integral of derivative absorption spectra; $\mathrm{B} \perp \mathrm{c}$-axis) of the total As spectrum. The dashed line represents a least-square linear fitting of the inverse signal intensity versus $T$ demonstrating a paramagnetic $\left(\chi \cdot \propto \cdot\left(T \cdot-\cdot T_{c}\right)-1\right)$ behavior with a close to zero Curie-Weiss temperature $\mathrm{Tc}_{\mathrm{c}}=\cdot-1 \cdot \pm \cdot 1 \mathrm{~K}$. The error bars indicate the uncertainty reflected in the data sets. 5 Semicond. Sci. Technol. 34 (2019) 035022 B Schoenaers et al decrease of the 75As hf quartet on the total intensity, accentuating the importance of taking into account only the four-peak hf part of the total spectrum to reliably determine the acceptor activation energy corresponding to decoupled dopants; as known from ESR insight, such (primary) hf structure reflects electron-nuclear interaction at isolated impurities doping in the desired 'ideal' decoupled manner. Pertinently, we may add here that for the case of the above P-in-Si study, it is also the intensity of the hf component of the ${ }_{31} \mathrm{P}$ ESR signal (in this case, naturally only being composed of hf parts) that has been monitored versus $T$ to correctly infer $\mathrm{E}_{\mathrm{a}}(\mathrm{P})$. 
Obviously, on statistical grounds, the chance for clustering will rise with increasing impurity content, the more so for systems out of equilibrium. Yet, while part of the relatively large fraction of As cluster formation may have been rendered by the encountered, rather high, concentration of As impurities, the bunching is likely more tied to the history and modalities (i.e. temperatures, ambient, dispersion, etc) of the sample formation over geological periods, beset with growth irregularity and inhomogeneity. In the latter view, it can thus not be excluded that, even in geo-MoS2 of asincorporated significantly lower As impurity concentration, there may still be present a considerable fraction of As clustering. Deeper insight on this may be gained from a comparative ESR study on a set of synthetic MoS2 specimen controllably doped by As to desired different concentrations.

4. Conclusions

In summary, the As acceptor emerges as a quite robust dopant of bulk geological $\mathrm{MoS}_{2}$ as it sustains annealing in neutral ambient (vacuum) up to $840{ }^{\circ} \mathrm{C}$. When subjected to sequential 'harsh' thermal treatments in $\mathrm{H} 2$ up to this $\mathrm{T}$, some decrease in As acceptor density is observed, but not nearly as drastic as for the $\mathrm{N}$ acceptor in synthetic MoS2. Moreover, the As acceptor signal intensity could only be partially recovered at $840^{\circ} \mathrm{C}$ in vacuum.

As to its dopant properties, the previously obtained As acceptor activation energy of $0.7 \cdot \pm \cdot 0.2 \mathrm{meV}$ at K-band, corresponding to decoupled dopants, has been firmly consolidated by X-band observations. The total As signal on the other hand exhibits a close to zero Curie-Weiss temperature with no appreciable influence of dopant activation. The validity of the applied interpretation approach is firmly consolidated by a first activation energy assessment by ESR of $P$ dopants in $\mathrm{n}-\mathrm{Si}$, demonstrating an excellent agreement with the commonly accepted activation energy for decoupled $\mathrm{P}$ donors in Si.

In general, the current work firmly establishes As as utmost appropriate for covalently bonded p-type doping of $\mathrm{MoS}_{2}$ both in terms of its excellent thermal stability and appropriate activation energy.

ORCID iDs

A Stesmans https://orcid.org/0000-0001-8534-4475

\section{References}

[1] Novoselov K S, Geim A K, Morozov S V, Jiang D, Zhang Y, Dubonos S V, Grigorieva I V and Firsov A A 2004 Science 306666

[2] Wang Q H, Kalantar-Zadeh K, Kis A, Coleman J N and Strano M S 2012 Nat. Nanotechnol. 7699

[3] Voiry D, Mohite A and Chhowalla M 2015 Chem. Soc. Rev. 442702

[4] Radisavljevic B, Radenovic A, Brivio J, Giacometti V and

Kis A 2011 Nat. Nanotechnol. 6147

[5] Song I, Parkab C and Choi H C 2015 RSC Adv. 57495

[6] McDonnell S, Addou R, Hinkle C L and Wallace R M 2016 2D

Materials for Nanoelectronics ed M Houssa et al (London:

Taylor and Francis) p 163 ISBN: 978-1-4987-0417-5 
[7] Castro Neto A H and Novoselov K 2011 Mater. Express 110

[8] Song J-G et al 2015 Nat. Commun. 67817

[9] Geim A K and Grigorieva I V 2013 Nature 499419

[10] Ganatra R and Zhang Q 2014 ACS Nano 84074

[11] Cui X et al 2015 Nat. Nano 10534

[12] Schoenaers B, Stesmans A and Afanas'ev V V 2018 J. Vac.

Sci. Technol. A 3605 G503

[13] Brandao E D, Ribeiro G M, Vaz P H, González J C and

Krambrock K 2016 J. Appl. Phys. 119235701

[14] Title R S and Shafer M W 1972 Phys. Rev. Lett. 28808

[15] Title R S and Shafer M W 1973 Phys. Rev. B 8615

[16] Stesmans A, Iacovo S and Afanas'ev V V 2016 Appl. Phys.

Lett. 109172104

[17] Schoenaers B, Stesmans A and Afanas'ev V V 2017 AIP Adv. 7105006

[18] Schoenaers B, Stesmans A and Afanas'ev V V 2017 Phys.

Status Solidi C 141700211

[19] Iacovo S, Stesmans A, Houssa M and Afanas'ev V V 2017

J. Phys.: Condens. Matter 29 08LT01

[20] Sze S 1981 Physics of Semiconductor Devices (New York:

Wiley) p 21 ISBN: 0471842907

[21] SPI Supplies West Chester, USA

[22] Stesmans A 1988 J. Magn. Reson. 76 14-21

[23] Stesmans A and Wu Y 1988 J. Phys. D: Appl. Phys. 211205

[24] Weil J A, Bolton J R and Wertz J E 1994 Electron Paramagnetic

Resonance: Elementary Theory and Practical applications

(New York: Wiley) pp 504-7 ISBN: 0-471-57234-9

[25] Stesmans A 1993 Phys. Rev. B 482418

[26] Pankove J I, Carlson D E, Berkeyheiser J E and Wance R O

1983 Phys. Rev. Lett. 512224

[27] Stesmans A 2000 Phys. Rev. B 618393

[28] Blakemore J S 1962 Semiconductor Statistics (Oxford:

Pergamon) 978-0486495026

[29] Scalise E, Houssa M, Pourtois G, Afanas'ev V V and

Stesmans A 2014 Physica E 56416

Scalise E 2016 2D Materials for Nanoelectronics ed M Houssa

et al (London: Taylor and Francis) p 141 ISBN: 978-1-4987-

0417-5

[30] Cheiwchanchamnangij T and Lambrecht W 2012 Phys. Rev. B

85205302

[31] Suh J et al 2014 Nano Lett. 146976

[32] Feher G, Fletcher R C and Gere E A 1955 Phys. Rev. 1001784

[33] Ue H and Maekawa S 1971 Phys. Rev. B 34232

Quirt J D and Marko J R 1973 Phys. Rev. B 73842

6 